Research Article

\title{
Some Properties of a Sequence Similar to Generalized Euler Numbers
}

\section{Haiqing Wang and Guodong Liu}

Department of Mathematics, Huizhou University, Huizhou, Guangdong 516007, China

Correspondence should be addressed to Guodong Liu; gdliu@pub.huizhou.gd.cn

Received 27 December 2012; Accepted 7 February 2013

Academic Editors: W. F. Klostermeyer, T. Prellberg, S. Rim, and W. F. Smyth

Copyright (C) 2013 H. Wang and G. Liu. This is an open access article distributed under the Creative Commons Attribution License, which permits unrestricted use, distribution, and reproduction in any medium, provided the original work is properly cited.

We introduce the sequence $\left\{U_{n}^{(x)}\right\}$ given by generating function $\left(1 /\left(e^{t}+e^{-t}-1\right)\right)^{x}=\sum_{n=0}^{\infty} U_{n}^{(x)}\left(t^{n} / n !\right)\left(|t|<(1 / 3) \pi, 1^{x}:=1\right)$ and establish some explicit formulas for the sequence $\left\{U_{n}^{(x)}\right\}$. Several identities involving the sequence $\left\{U_{n}^{(x)}\right\}$, Stirling numbers, Euler polynomials, and the central factorial numbers are also presented.

\section{Introduction and Definitions}

For a real or complex parameter $\alpha$, the generalized Euler polynomials $E_{n}^{(\alpha)}(x)$ are defined by the following generating function (see [1-4])

$$
\left(\frac{2}{e^{t}+1}\right)^{\alpha} e^{x t}=\sum_{n=0}^{\infty} E_{n}^{(\alpha)}(x) \frac{t^{n}}{n !} \quad\left(|t|<\pi, 1^{\alpha}:=1\right) .
$$

Obviously, we have

$$
E_{n}^{(1)}(x)=E_{n}(x) \quad\left(n \in \mathbb{N}_{0}:=\mathbb{N} \cup\{0\}\right),
$$

in terms of the classical Euler polynomials $E_{n}(x), \mathbb{N}$ being the set of positive integers. The classical Euler numbers $E_{n}$ are given by the following:

$$
E_{n}=2^{n} E_{n}\left(\frac{1}{2}\right) \quad\left(n \in \mathbb{N}_{0}\right) .
$$

The so-called the generalized Euler numbers $E_{2 n}^{(x)}$ are defined by $($ see $[3,5])$

$$
\left(\frac{2}{e^{t}+e^{-t}}\right)^{x}=\sum_{n=0}^{\infty} E_{2 n}^{(x)} \frac{t^{2 n}}{(2 n) !} \quad\left(|t|<\frac{\pi}{2}, 1^{x}:=1\right) .
$$

In fact, $E_{2 n}^{(k)}(k \in \mathbb{Z})$ are the Euler numbers of order $k, \mathbb{Z}$ being the set of integers. The numbers $E_{2 n}^{(1)}=E_{2 n}$ are the ordinary Euler numbers.
Zhi-Hong Sun introduces the sequence $\left\{U_{n}\right\}$ similar to Euler numbers as follows (see $[6,7])$ :

$$
U_{0}=1, \quad U_{n}=-2 \sum_{k=1}^{[n / 2]}\left(\begin{array}{c}
n \\
2 k
\end{array}\right) U_{n-2 k}, \quad(n \geq 1),
$$

where (and in what follows) $[x]$ is the greatest integer not exceeding $x$.

Clearly, $U_{2 n-1}=0$ for $n \geq 1$. The first few values of $U_{2 n}$ are shown below

$$
\begin{gathered}
U_{2}=-2, \quad U_{4}=22, \quad U_{6}=-602, \quad U_{8}=30742, \\
U_{10}=-2523002, \quad U_{12}=303692662 .
\end{gathered}
$$

The sequence $\left\{U_{n}\right\}$ is related to the classical Bernoulli polynomials $B_{n}(x)$ (see [8-11]) and the classical Euler polynomials $E_{n}(x)$. Zhi-Hong Sun gets the generating function of 
$\left\{U_{n}\right\}$ and deduces many identities involving $\left\{U_{n}\right\}$. As example, (see [6]),

$$
\begin{aligned}
& \frac{1}{e^{t}+e^{-t}-1}=\sum_{n=0}^{\infty} U_{n} \frac{t^{n}}{n !} \\
&=\sum_{n=0}^{\infty} U_{2 n} \frac{t^{2 n}}{(2 n) !} \quad\left(|t|<\frac{1}{3} \pi\right), \\
& \frac{1}{2 \cos t-1}= \sum_{n=0}^{\infty}(-1)^{n} U_{2 n} \frac{t^{2 n}}{(2 n) !} \quad\left(|t|<\frac{1}{3} \pi\right), \\
& U_{2 n}=3^{2 n} E_{2 n}\left(\frac{1}{3}\right) .
\end{aligned}
$$

Similarly, we can define the generalized sequence $\left\{U_{n}^{(x)}\right\}$. For a real or complex parameter $x$, the generalized sequence $\left\{U_{n}^{(x)}\right\}$ is defined by the following generating function:

$$
\left(\frac{1}{e^{t}+e^{-t}-1}\right)^{x}=\sum_{n=0}^{\infty} U_{n}^{(x)} \frac{t^{n}}{n !} \quad\left(|t|<\frac{1}{3} \pi, 1^{x}:=1\right) .
$$

Obviously,

$$
U_{0}^{(x)}=1, \quad U_{n}^{(1)}=U_{n} \quad(n \in \mathbb{N}) .
$$

By using (10), we can obtain

$$
U_{n}^{(k)}=n ! \sum_{v_{1}, \ldots, v_{k} \in \mathbb{N}_{0}}^{\left(v_{1}+\cdots+v_{k}=n\right)} \frac{U_{v_{1}} \cdots U_{v_{k}}}{v_{1} ! \cdots v_{k} !} \quad(k \in \mathbb{N}) .
$$

We now return to the Stirling numbers $s(n, k)$ of the first kind, which are usually defined by (see $[2,5,8,11,12])$

$$
x(x-1)(x-2) \cdots(x-n+1)=\sum_{k=0}^{n} s(n, k) x^{k}
$$

or by the following generating function:

$$
(\log (1+x))^{k}=k ! \sum_{n=k}^{\infty} s(n, k) \frac{x^{n}}{n !} .
$$

It follows from (13) or (14) that

$$
s(n, k)=s(n-1, k-1)-(n-1) s(n-1, k)
$$

and that

$$
\begin{gathered}
s(n, 0)=0 \quad(n \in \mathbb{N}), \quad s(n, n)=1 \quad\left(n \in \mathbb{N}_{0}\right), \\
s(n, 1)=(-1)^{n-1}(n-1) ! \quad(n \in \mathbb{N}), \\
s(n, k)=0 \quad(k>n \text { or } k<0) .
\end{gathered}
$$

The central factorial numbers $T(n, k)$ are given by the following expansion formula (see $[3,5,13])$ :

$$
\begin{aligned}
x^{n}= & \sum_{k=0}^{n} T(n, k) x\left(x-1^{2}\right) \\
& \quad \times\left(x-2^{2}\right) \cdots\left(x-(k-1)^{2}\right)
\end{aligned}
$$

or by means of the generating function

$$
\left(e^{x}+e^{-x}-2\right)^{k}=(2 k) ! \sum_{n=k}^{\infty} T(n, k) \frac{x^{2 n}}{(2 n) !} .
$$

It follows from (17) or (18) that

$$
T(n, k)=T(n-1, k-1)+k^{2} T(n-1, k),
$$

with

$$
\begin{gathered}
T(0,0)=1, \quad T(n, 0)=0 \quad(n \in \mathbb{N}), \\
T(n, 1)=1 \quad(n \in \mathbb{N}) .
\end{gathered}
$$

We also find from (18) that

$$
\begin{gathered}
T(n, 2)=\frac{1}{4}\left(4^{n-1}-1\right), \\
T(n, 3)=\frac{9^{n}}{360}-\frac{4^{n}}{60}+\frac{1}{24} \quad(n \in \mathbb{N}) .
\end{gathered}
$$

The main purpose of this paper is to prove some formulas for the generalized sequence $\left\{U_{n}^{(x)}\right\}$ and $E_{n}(x)$. Some identities involving the sequence $\left\{U_{n}^{(x)}\right\}$, Stirling numbers $s(n, k)$, and the central factorial numbers $T(n, k)$ are deduced.

\section{Main Results}

Theorem 1. Let $n \geq k(n, k \in \mathbb{N})$ and

$$
q(n, k)=(-1)^{k} \sum_{j=k}^{n} \frac{(2 j) !}{j !} T(n, j) s(j, k) .
$$

Then,

$$
U_{2 n}^{(x)}=\sum_{k=1}^{n} q(n, k) x^{k}
$$

Remark 2. By (15), (19), (20), and Theorem 1, we know that $U_{2 n}^{(x)}$ is a polynomial of $x$ with integral coefficients. For example, by setting $n=1,2,3,4$ in Theorem 1 , we get

$$
\begin{gathered}
U_{2}^{(x)}=-2 x, \quad U_{4}^{(x)}=10 x+12 x^{2}, \\
U_{6}^{(x)}=-182 x-300 x^{2}-120 x^{3}, \\
U_{8}^{(x)}=6970 x+13692 x^{2}+8400 x^{3}+1680 x^{4} .
\end{gathered}
$$

Taking $x=1$ in Theorem 1, we can obtain the following.

Corollary 3. Let $n \in \mathbb{N}$. Then,

$$
U_{2 n}=\sum_{j=0}^{n}(-1)^{j}(2 j) ! T(n, j) .
$$

From Corollary 3, we may immediately deduce the following results. 
Corollary 4. Let $n \in \mathbb{N}$. Then,

$$
\begin{gathered}
U_{2 n} \equiv-2(\bmod 24), \\
U_{2 n} \equiv-2+24 T(n, 2)(\bmod 720), \\
U_{2 n} \equiv-2+24 T(n, 2)-720 T(n, 3)(\bmod 40320) .
\end{gathered}
$$

Theorem 5. Let $n \geq k(n, k \in \mathbb{N})$. Then,

$$
\begin{gathered}
U_{2 n}=\sum_{k=1}^{n} q(n, k), \\
U_{2 n}=2 \sum_{k=1}^{[n / 2]} q(n, 2 k)-2 \\
=2 \sum_{k=1}^{[(n-1) / 2]} q(n, 2 k+1)+2 .
\end{gathered}
$$

Theorem 6. Let $n \geq k(n, k \in \mathbb{N})$. Suppose also that $q(n, k)$ is defined by (22). Then,

$$
\begin{gathered}
k ! q(n, k)=(2 n) ! 3^{2 n-k} \\
\times \sum_{v_{1}, \ldots, v_{k} \in \mathbb{N}}^{\left(v_{1}+\cdots+v_{k}=n\right)}\left(E_{2 v_{1}-1}(0)-E_{2 v_{1}-1}\left(\frac{2}{3}\right)\right) \\
\cdots\left(E_{2 v_{k}-1}(0)-E_{2 v_{k}-1}\left(\frac{2}{3}\right)\right) \\
\times\left(\left(2 v_{1}\right) ! \cdots\left(2 v_{k}\right) !\right)^{-1} .
\end{gathered}
$$

Theorem 7. Let $n \in \mathbb{N}$. Then,

$$
-2 \sum_{k=0}^{n-1}\left(\begin{array}{c}
2 n-1 \\
2 k
\end{array}\right) U_{2 k}=3^{2 n-1}\left(E_{2 n-1}(0)-E_{2 n-1}\left(\frac{2}{3}\right)\right) .
$$

Theorem 8. Let $n \in \mathbb{N}$. Then,

$$
U_{n+1}=\sum_{k=0}^{n-1}\left(\begin{array}{l}
n \\
k
\end{array}\right)\left(\left(1-2^{n-k}\right) U_{k+1}-2^{n-k} U_{k}\right)
$$

Theorem 9. Let $n \in \mathbb{N}_{0}$. Then,

$$
\sum_{n=0}^{\infty} \frac{1}{(n+1) !} U_{n}=\frac{1}{\sqrt{3}} \log \frac{2 e-1-\sqrt{3}}{2(2-\sqrt{3}) e-5+3 \sqrt{3}} .
$$

\section{Proofs of Theorems}

Proof of Theorem 1. By (10), (13), and (18), we have

$$
\begin{aligned}
\sum_{n=0}^{\infty} U_{2 n}^{(x)} \frac{t^{2 n}}{(2 n) !} & =\left(\frac{1}{e^{t}+e^{-t}-1}\right)^{x} \\
& =\left(\frac{1}{1+\left(e^{t}+e^{-t}-2\right)}\right)^{x} \\
& =\sum_{j=0}^{\infty}(-1)^{j}\left(\begin{array}{c}
x+j-1 \\
j
\end{array}\right)\left(e^{t}+e^{-t}-2\right)^{j} \\
& =\sum_{j=0}^{\infty}(-1)^{j}\left(\begin{array}{c}
x+j-1 \\
j
\end{array}\right)(2 j) ! \sum_{n=j}^{\infty} T(n, j) \frac{t^{2 n}}{(2 n) !} \\
& =\sum_{n=0}^{\infty} \frac{t^{2 n}}{(2 n) !} \sum_{j=0}^{n}(-1)^{j}(2 j) !\left(\begin{array}{c}
x+j-1 \\
j
\end{array}\right) T(n, j),
\end{aligned}
$$

which readily yields

$$
\begin{aligned}
U_{2 n}^{(x)} & =\sum_{j=0}^{n}(-1)^{j}(2 j) !\left(\begin{array}{c}
x+j-1 \\
j
\end{array}\right) T(n, j) \\
& =\sum_{j=0}^{n}(-1)^{j}(2 j) ! T(n, j) \frac{1}{j !} x(x+1) \cdots(x+j-1) \\
& =\sum_{j=0}^{n} \frac{(2 j) !}{j !} T(n, j) \sum_{k=1}^{j}(-1)^{k} s(j, k) x^{k} \\
& =\sum_{k=1}^{n}(-1)^{k} \sum_{j=k}^{n} \frac{(2 j) !}{j !} T(n, j) s(j, k) x^{k} \\
& =\sum_{k=1}^{n} q(n, k) x^{k} .
\end{aligned}
$$

This completes the proof of Theorem 1 .

Proof of Theorem 5. By (10), we have

$$
\sum_{n=0}^{\infty} U_{2 n}^{(-1)} \frac{t^{2 n}}{(2 n) !}=e^{t}+e^{-t}-1=2 \sum_{n=0}^{\infty} \frac{t^{2 n}}{(2 n) !}-1
$$

and $U_{0}^{(x)}=1$, thus

$$
\sum_{n=1}^{\infty} U_{2 n}^{(-1)} \frac{t^{2 n}}{(2 n) !}=e^{t}+e^{-t}-1=2 \sum_{n=1}^{\infty} \frac{t^{2 n}}{(2 n) !}
$$

By Theorem 1 and comparing the coefficient of $t^{2 n} /(2 n)$ ! on both sides of (35), we get

$$
\sum_{k=1}^{n} q(n, k)(-1)^{k}=U_{2 n}^{(-1)}=2
$$


Again, by taking $x=1$ in Theorem 1, we have

$$
\sum_{k=1}^{n} q(n, k)=U_{2 n}
$$

By (36) and (37), we immediately obtain (27). This completes the proof of Theorem 5 .

Proof of Theorem 6. By applying Theorem 1, we have

$$
k ! q(n, k)=\left.\frac{d^{k}}{d x^{k}}\left\{U_{n}^{(x)}\right\}\right|_{x=0}
$$

On the other hand, it follows from (10) that

$$
\left.\sum_{n=k}^{\infty} \frac{d^{k}}{d x^{k}}\left\{U_{n}^{(x)}\right\}\right|_{x=0} \frac{t^{2 n}}{(2 n) !}=\left(\log \left(\frac{1}{e^{t}+e^{-t}-1}\right)\right)^{k}
$$

By using (38) and (39), we find that

$$
k ! \sum_{n=k}^{\infty} q(n, k) \frac{t^{2 n}}{(2 n) !}=\left(\log \left(\frac{1}{e^{t}+e^{-t}-1}\right)\right)^{k} .
$$

We now note that

$$
\begin{aligned}
\frac{d}{d t}\left\{\log \left(\frac{1}{e^{t}+e^{-t}-1}\right)\right\} \\
=\frac{e^{-t}-e^{t}}{e^{t}+e^{-t}-1} \\
=\frac{e^{-t}-e^{t}}{2}\left(\frac{2 e^{t}}{e^{3 t}+1}+\frac{2 e^{-t}}{e^{-3 t}+1}\right) \\
=\frac{1}{2}\left(\left(\frac{2}{e^{3 t}+1}-\frac{2}{e^{-3 t}+1}\right)-\left(\frac{2 e^{2 t}}{e^{3 t}+1}-\frac{2 e^{-2 t}}{e^{-3 t}+1}\right)\right) \\
=\frac{1}{2}\left(\sum_{n=0}^{\infty} E_{n}(0) \frac{(3 t)^{n}}{n !}-\sum_{n=0}^{\infty} E_{n}(0) \frac{(-3 t)^{n}}{n !}\right) \\
-\frac{1}{2}\left(\sum_{n=0}^{\infty} E_{n}\left(\frac{2}{3}\right) \frac{(3 t)^{n}}{n !}-\sum_{n=0}^{\infty} E_{n}\left(\frac{2}{3}\right) \frac{(-3 t)^{n}}{n !}\right) \\
=\sum_{n=0}^{\infty} 3^{2 n+1}\left(E_{2 n+1}(0)-E_{2 n+1}\left(\frac{2}{3}\right)\right) \frac{t^{2 n+1}}{(2 n+1) !} .
\end{aligned}
$$

Hence,

$$
\begin{aligned}
\log \frac{1}{e^{t}+e^{-t}-1} & =\sum_{n=0}^{\infty} 3^{2 n+1}\left(E_{2 n+1}(0)-E_{2 n+1}\left(\frac{2}{3}\right)\right) \frac{t^{2 n+2}}{(2 n+2) !} \\
& =\sum_{n=1}^{\infty} 3^{2 n-1}\left(E_{2 n-1}(0)-E_{2 n-1}\left(\frac{2}{3}\right)\right) \frac{t^{2 n}}{(2 n) !}
\end{aligned}
$$

yields

$$
\begin{aligned}
& k ! \sum_{n=k}^{\infty} q(n, k) \frac{t^{2 n}}{(2 n) !} \\
& =\left(\sum_{n=1}^{\infty} 3^{2 n-1}\left(E_{2 n-1}(0)-E_{2 n-1}\left(\frac{2}{3}\right)\right) \frac{t^{2 n}}{(2 n) !}\right)^{k} \\
& =\sum_{n=k}^{\infty} \frac{t^{2 n}}{(2 n) !}(2 n) ! 3^{2 n-k} \\
& \quad \times \sum_{v_{1}, \ldots, v_{k} \in \mathbb{N}}^{\left(v_{1}+\cdots+v_{k}=n\right)}\left(E_{2 v_{1}-1}(0)-E_{2 v_{1}-1}\left(\frac{2}{3}\right)\right) \\
& \quad \times\left(\left(2 v_{1}\right) ! \cdots\left(2 v_{k}\right) !\right)^{-1} .
\end{aligned}
$$

Comparing the coefficient of $t^{2 n} /(2 n)$ ! on both sides of (43), we immediately get (28). This completes the proof of Theorem 6 .

Proof of Theorem 7. Consider

$$
\begin{aligned}
\frac{d}{d t}\left\{\log \left(\frac{1}{e^{t}+e^{-t}-1}\right)\right\} & =\frac{e^{-t}-e^{t}}{e^{t}+e^{-t}-1} \\
& =\sum_{n=0}^{\infty} U_{2 n} \frac{t^{2 n}}{(2 n) !}\left(-2 \sum_{n=0}^{\infty} \frac{t^{2 n+1}}{(2 n+1) !}\right) \\
& =-2 \sum_{n=0}^{\infty} \sum_{k=0}^{n}\left(\begin{array}{c}
2 n+1 \\
2 k
\end{array}\right) U_{2 k} \frac{t^{2 n+1}}{(2 n+1) !}
\end{aligned}
$$

Thus,

$$
\log \frac{1}{e^{t}+e^{-t}-1}=-2 \sum_{n=1}^{\infty} \sum_{k=0}^{n-1}\left(\begin{array}{c}
2 n-1 \\
2 k
\end{array}\right) U_{2 k} \frac{t^{2 n}}{(2 n) !} .
$$

By (42) and (45) we obtain (29). This completes the proof of Theorem 7.

Proof of Theorem 8. By using (7), we have

$$
\sum_{n=1}^{\infty} U_{n} \frac{t^{n-1}}{(n-1) !}=\frac{e^{-t}-e^{t}}{\left(e^{t}+e^{-t}-1\right)^{2}} .
$$

Thus

$$
\begin{gathered}
\left(e^{2 t}-e^{t}+1\right) \sum_{n=1}^{\infty} U_{n} \frac{t^{n-1}}{(n-1) !}=\left(1-e^{2 t}\right) \sum_{n=0}^{\infty} U_{n} \frac{t^{n}}{n !}, \\
\sum_{n=0}^{\infty}\left(2^{n}-1\right) \frac{t^{n}}{n !} \sum_{n=0}^{\infty} U_{n+1} \frac{t^{n}}{n !}+\sum_{n=0}^{\infty} U_{n+1} \frac{t^{n}}{n !} \\
=\sum_{n=0}^{\infty} U_{n} \frac{t^{n}}{n !}-\sum_{n=0}^{\infty} 2^{n} \frac{t^{n}}{n !} \sum_{n=0}^{\infty} U_{n} \frac{t^{n}}{n !} .
\end{gathered}
$$


That is,

$$
\begin{gathered}
\sum_{n=0}^{\infty} \sum_{k=0}^{n}\left(\begin{array}{l}
n \\
k
\end{array}\right)\left(2^{n-k}-1\right) U_{k+1} \frac{t^{n}}{n !}+\sum_{n=0}^{\infty} U_{n+1} \frac{t^{n}}{n !} \\
=\sum_{n=0}^{\infty} U_{n} \frac{t^{n}}{n !}-\sum_{n=0}^{\infty} \sum_{k=0}^{n}\left(\begin{array}{l}
n \\
k
\end{array}\right) 2^{n-k} U_{k} \frac{t^{n}}{n !}
\end{gathered}
$$

Comparing the coefficient of $t^{n} / n$ ! on both sides of (48), we get the following:

$$
U_{n+1}-U_{n}=\sum_{k=0}^{n}\left(\begin{array}{l}
n \\
k
\end{array}\right)\left(\left(1-2^{n-k}\right) U_{k+1}-2^{n-k} U_{k}\right) .
$$

By (49) we immediately obtain (30). This completes the proof of Theorem 8.

Proof of Theorem 9. By integrating (7) with respect to $t$ from 0 to 1 , we have

$$
\begin{aligned}
\sum_{n=0}^{\infty} \frac{1}{(n+1) !} U_{n} & =\int_{0}^{1} \frac{1}{e^{t}+e^{-t}-1} d t \\
& =\int_{0}^{1} \frac{1}{e^{2 t}-e^{t}+1} d e^{t}=\int_{1}^{e} \frac{1}{x^{2}-x+1} d x .
\end{aligned}
$$

By $(50)$ and $\int\left(1 /\left(a x^{2}+b x+c\right)\right) d x=\left(1 / \sqrt{b^{2}-4 a c}\right) \log \mid(2 a x+$ $\left.b-\sqrt{b^{2}-4 a c}\right) /\left(2 a x+b+\sqrt{b^{2}-4 a c}\right) \mid+c$ ( $c$ is constant $)$, we have (31). This completes the proof of Theorem 9 .

\section{Acknowledgments}

This work is partly supported by the Social Science Foundation (no. 2012YB03) of Huizhou University and the Key Discipline Foundation (no. JG2011019) of Huizhou University.

\section{References}

[1] G. D. Liu, "On congruences of Euler numbers modulo powers of two," Journal of Number Theory, vol. 128, no. 12, pp. 3063-3071, 2008.

[2] G. D. Liu and H. M. Srivastava, "Explicit formulas for the Nörlund polynomials $B_{n}^{(x)}$ and $b_{n}^{(x)}$," Computers \& Mathematics with Applications, vol. 51, no. 9-10, pp. 1377-1384, 2006.

[3] G. D. Liu and W. P. Zhang, "Applications of an explicit formula for the generalized Euler numbers," Acta Mathematica Sinica, vol. 24, no. 2, pp. 343-352, 2008.

[4] Y. L. Luke, The Special Functions and Their Approximations, Vol. I, Academic Press, New York, NY, USA, 1969.

[5] H. M. Srivastava and G. D. Liu, "Some identities and congruences involving a certain family of numbers," Russian Journal of Mathematical Physics, vol. 16, no. 4, pp. 536-542, 2009.

[6] Z. H. Sun, "Identities and congruences for a new sequence," International Journal of Number Theory, vol. 8, no. 1, pp. 207225, 2012.

[7] Z. H. Sun, "Some properties of a sequence analogous to Euler numbers," Bulletin of the Australian Mathematical Society, 2012.
[8] C. Jordan, Calculus of Finite Differences, Chelsea, New York, NY, USA, 1965.

[9] G. D. Liu, "The D numbers and the central factorial numbers," Publicationes Mathematicae Debrecen, vol. 79, no. 1-2, pp. 41-53, 2011.

[10] G. D. Liu, "A recurrence formula for $D$ numbers $D_{2 n}^{(2 n-1)}$ ", Discrete Dynamics in Nature and Society, Article ID 605313, 6 pages, 2009.

[11] N. E. Nörlund, Vorlesungen über Differenzenrechnung, Springer, Berlin, Germany, 1924, Reprinted by Chelsea, Bronx, New York, NY, USA, 1954.

[12] L. Comtet, Advanced Combinatorics, D. Reidel, Dordrecht, The Netherlands, 1974, (Translated from the French by J. W. Nienhuys).

[13] J. Riordan, Combinatorial Identities, John Wiley \& Sons, New York, NY, YSA, 1968. 


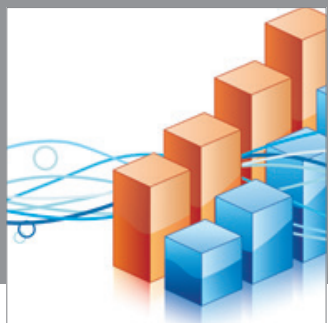

Advances in

Operations Research

mansans

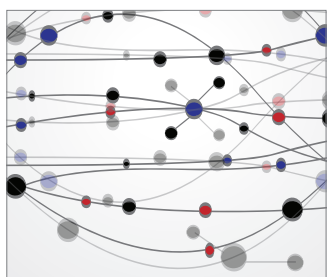

The Scientific World Journal
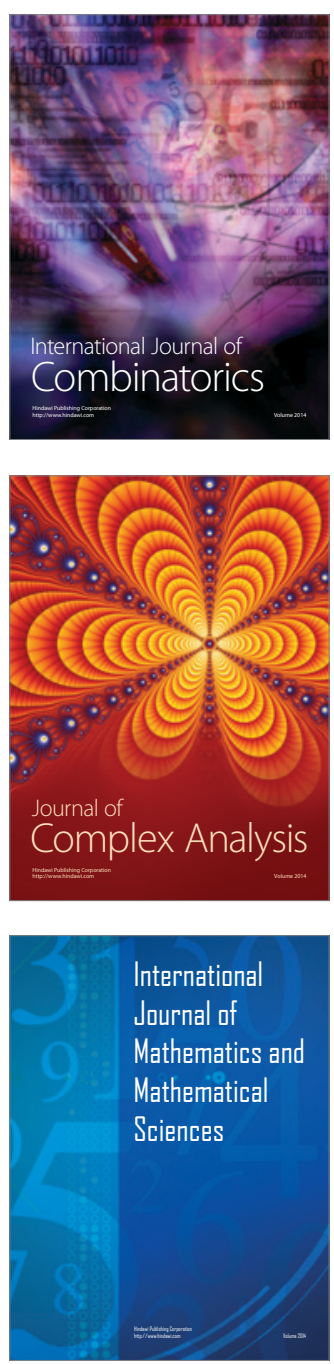
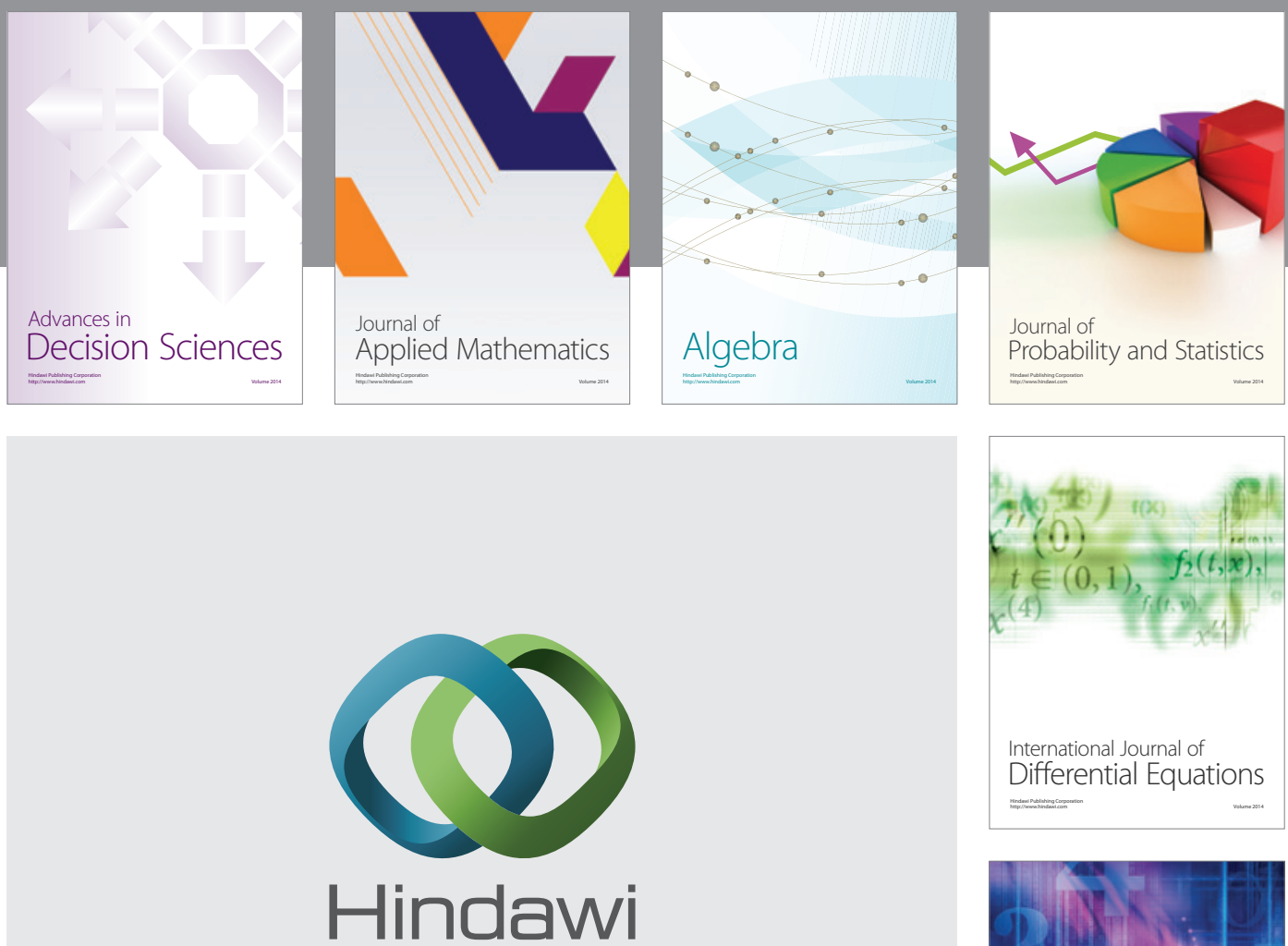

Submit your manuscripts at http://www.hindawi.com
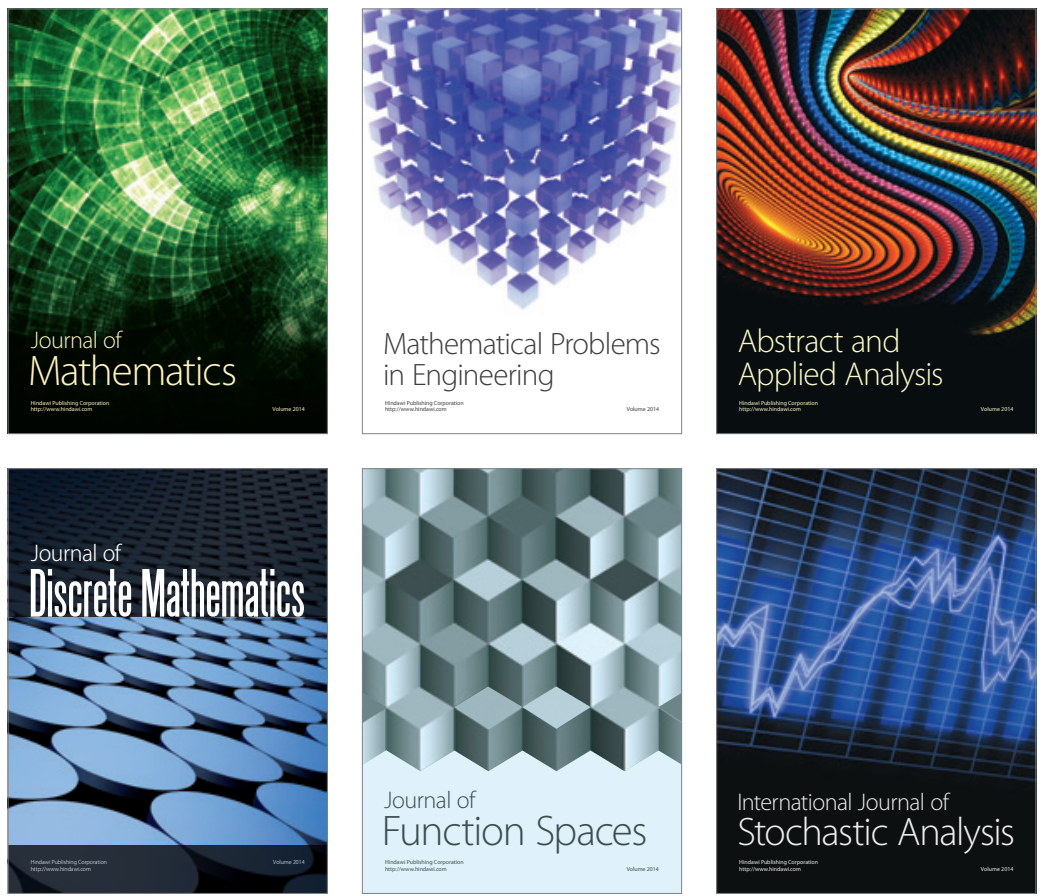

Journal of

Function Spaces

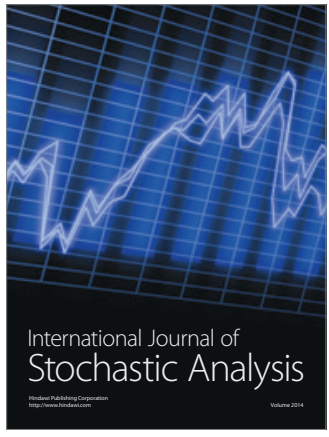

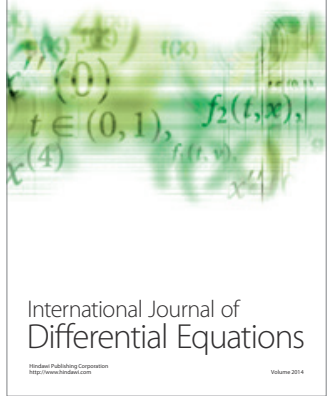
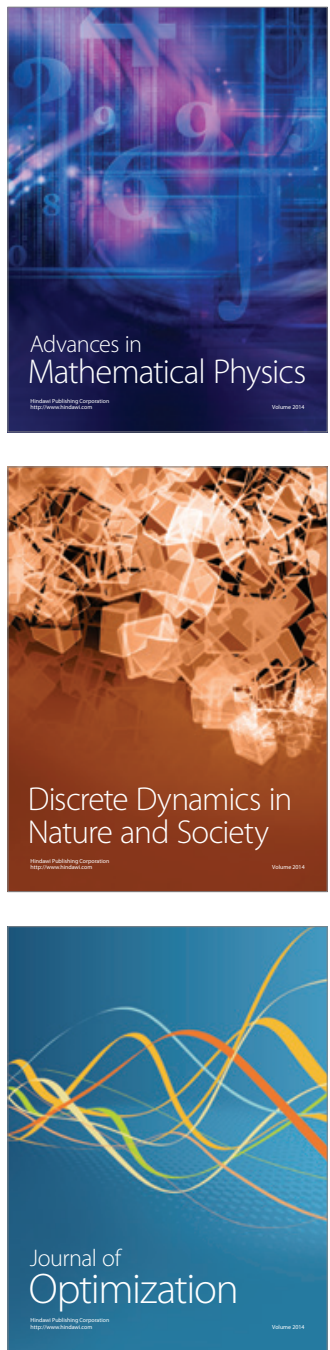